\title{
Exploitation and Research on 3D Digital Modeling of Track Structure Based on VBA
}

\author{
Feng HAN \\ Faculty of Geomatics \\ Lanzhou Jiaotong University \\ Lanzhou, China \\ e-mail: hanfeng740412@mail.lzjtu.cn
}

\author{
Feng KANG \\ School of Civil Engineering \\ Lanzhou Jiaotong University \\ Lanzhou, China \\ e-mail: 1540667369@qq.com
}

\begin{abstract}
The development of virtual simulation and BIM in railway industry has increasingly become a trend. The method of 3D digital modeling is important in design and construction, but the current study of track structure modeling is not accurate enough, and the species are not comprehensive. This thesis was based on the re-development of AutoCAD via VBA, Visual windows was designed for railway track components such as fastening, sleeper, and railway switch., and this paper discusses the method of parametric drawing design and establishing three-dimensional library via VBA language for track Structure. At the same time, custom menu is added on AutoCAD drawing window for connecting with programming, which realizes the automatic modeling of various types of track structure based on VBA, and Visual windows are friendly, three dimensional modeling of track components are simple and accurate. It has certain directive significance to building 3d model library and stronger practical value, which conforms to the development trend of railway industry.
\end{abstract}

Keywords-VBA; AutoCAD; track structure; parameterization; three dimensional modeling

\section{INTRODUCTION}

The railway development becomes information-based, automotive and intellectualized with the development of computer technology recently, and the design of railway becomes more and more mature. The real construction and design based on 3-D technology are more intuitive and it's speedier to manage its information compared with traditional 2D drawings. Firstly, it's necessary to get the information of the surface shape of each structure to build 3D model of the rail. Currently, there are many different methods to get the information of the surface shape and 3D grids, for example, using 3d laser scanning apparatus, editing the toolkit, buying models from business database and using modeling software, such as AutoCAD, OpenGL, 3DSMAX and so on. As is known to all, it's easy to make engineering drawings with plan figures and the rendered $3 \mathrm{~d}$ scenes by using AutoCAD, which is one of the most widely used software in railway.

But there isn't any 3d graphic file of railway structure internally, which results in heavy workload and too much repeated process when making some specialized $3 \mathrm{~d}$ graphics such as special fastening, sleeper, and railway switch , and it leads to a wasting of resources and a low efficiency.
However, AutoCAD's openness and rich development tools such as VBA, Visual LISP, ARX etc., provide effective means to solve this problem. AutoCAD VBA has more advantages compared with $\mathrm{ARX}$ based on $\mathrm{C} / \mathrm{C}++/ \mathrm{VC}$ programming language. It is easy for beginners to learn the grammar, not only that but VBA can provide a powerful function of creating forms, and it can implement access and management of Access database or other external databases by ActiveX Data Object with a quick execution and powerful function, therefore it has a extensive popularity. So this thesis tries to do some preliminary explorations and researches on the development of parameterized standard part $3 \mathrm{~d}$ library of railway structures under the guidance of object-oriented design thought.

\section{The SYSTEM OF TRACK STRUCTURE}

The track is one part of the railway and has a wide variety of structures. Each railway has corresponding track structure, so as to make sure that the train can run safety, smoothly and continuously under different conditions. The track are generally divided into two forms which are called ballasted track that contains rail, sleeper, connecting parts, ballast bed, anti-climbing equipment and railway switch, etc (as Fig.1), and ballastless track that contains rail, matching fasteners, bed board, adjusting layer of mortar, concrete base, etc , according to the different form of track structure (as Fig.2).

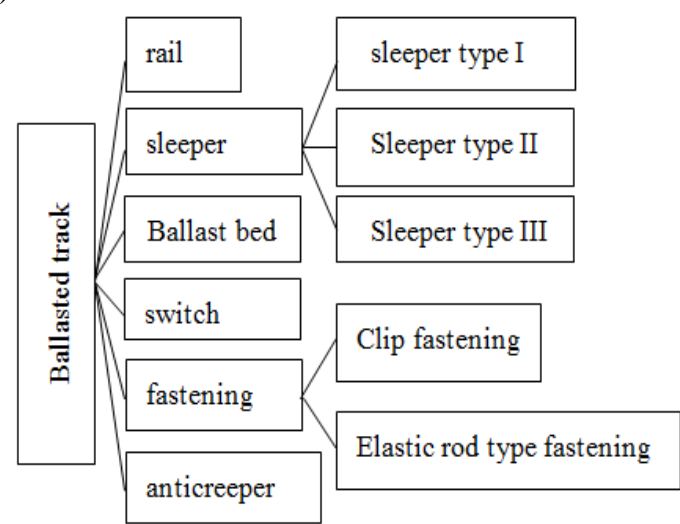

Figure 1. Ballasted track structure 


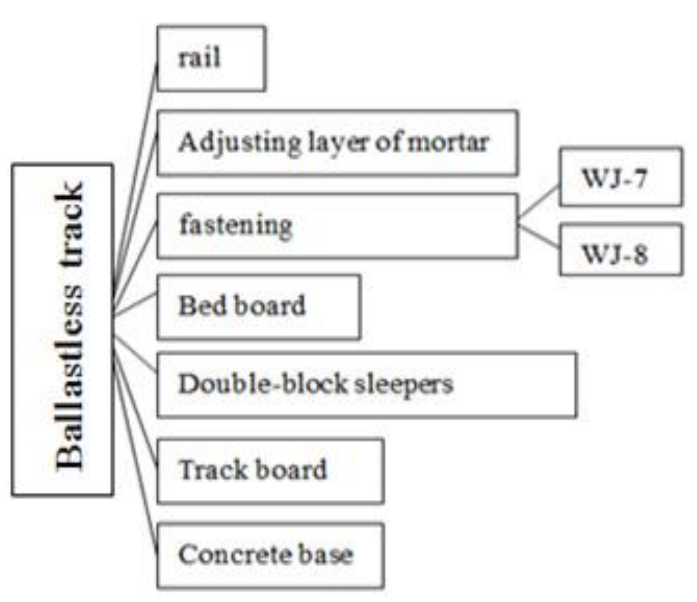

Figure 2. Ballastless track structure

\section{THE REALIZATION OF THE GRAPHICS PROGRAM}

\section{A. The Design of Visual Windows Based on VBA}

AutoCAD system for visual windows based on VBA is created, and the visual interface is designed correspondingly according the difference of track parts' structure. The repeated and computationally complex process will be complicated closely by the computer during the process of drawing. And all the designer need to do is inputting or choosing corresponding parameters. As a result, the working efficiency will be raised, the error caused by human will be avoided, and the accuracy will be improved, too. Taking the railway switch and the which are the most complex parts of the track structure as an example, the parameter like the length of switch rail, the radius of lead curve, the length of intermediate straight line and the heel length of frog can be imported manually with the difference of the railway switch number as is shown in the railway switch drawing windows in accordance with the up to date parameter list of railway switch as Fig.3, thus achieving the automatic drawing of the $3 \mathrm{~d}$ graphic railway switch for all types and the $3 \mathrm{~d}$ curve following turnout branch line according to different radius and central lines of track. These are of vital importance of 3d designing, construction and learning for the railway industry in the future.

Other parts like sleeper, fastening and track slab remain substantially constant because of complex structure, therefore there is no drawing interface of parameters inputting. The $3 \mathrm{~d}$ library is settled and the process of drawing will be encapsulated and finished automatically in the computer by programming. The drawing windows (shown in Fig.4 and Fig.5) are set for the purpose of a direct and convenient invocation for the designer.

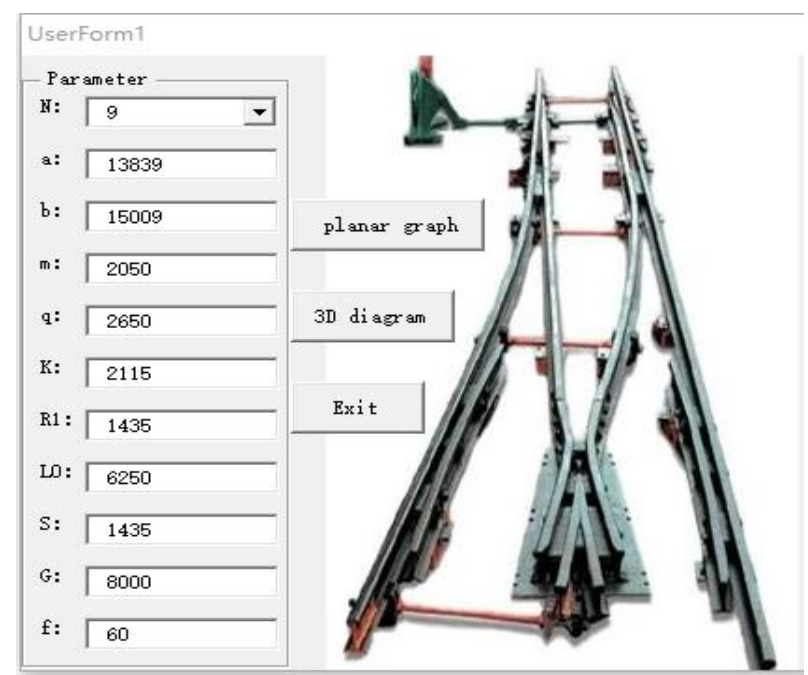

Figure 3. The diagram of parametric drawing window for railway switch

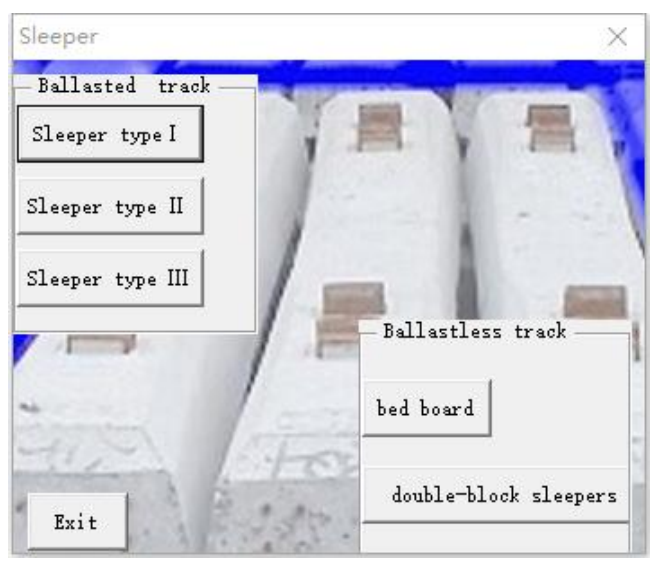

Figure 4. The diagram of drawing window for sleeper

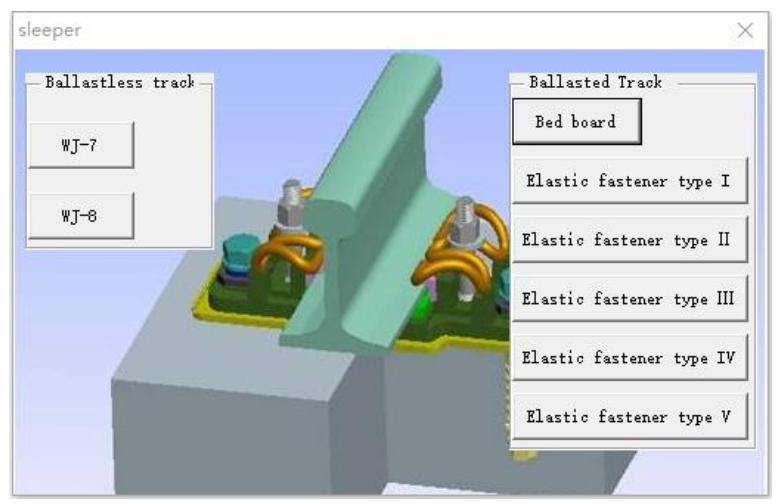

Figure 5. The diagram of drawing window for fastening

\section{B. The Design Thought and Program Invocation of VBA}

First of all, the visual drawing interface is designed, which is based on the analysis of the topology and the geometrical relationship. Then the parameter is needed to be added to the interface as required. Every parameter is defined and then the mathematical computer model can be researched. Afterwards, the computer can work out the 
abscissa and ordinate of the control point of each part in the track automatically after programming and debugging according to the VBA grammatical structures. The main program is shown below:

Dim zk As Double '

Dim jgc As Double '

Dim xjj As Double '

Dim gj As Double '

jgc $=$ TextBox8.Text

$\mathrm{xjj}=$ TextBox9.Text

$\mathrm{gj}=$ TextBox17.Text

$\mathrm{zk}=$ TextBox5.Text

Dim cc As Variant

cc = TDraw. Utility. Get Point (vbcrif \& "choose frog:")'

$\operatorname{pa}(0)=\operatorname{cc}(0)$

$\mathrm{pa}(1)=\mathrm{cc}(1)$

$\mathrm{pa}(2)=\mathrm{cc}(2)$

$\mathrm{yg}=\mathrm{gj}-\mathrm{zk} * \operatorname{Sin}(\mathrm{dcj})$

$\operatorname{po} 1(0)=\operatorname{pc}(0)-\mathrm{R} 1 * \operatorname{Sin}(\mathrm{dcj})-\mathrm{zk} * \operatorname{Cos}(\mathrm{dcj})$

$\operatorname{po} 1(1)=\operatorname{pd}(1)-\mathrm{R} 1 * \operatorname{Cos}(\mathrm{dcj})$

temp $=2 *(\mathrm{dcj}-\operatorname{Atn}(\mathrm{gj}-\mathrm{zk} * \operatorname{Sin}(\mathrm{dcj})-\mathrm{jgc} * \operatorname{Sin}($ beat $)) /$

$(\mathrm{qc}+\mathrm{hc}-\mathrm{zcgc}-\mathrm{jq}-\mathrm{jgc} * \operatorname{Cos}(\mathrm{beat})-\mathrm{zk} * \operatorname{Cos}($ beat $)))$

$\mathrm{t} 1=\mathrm{R} 1 *(\operatorname{Sin}($ temp $/ 2) / \operatorname{Cos}($ temp $/ 2))$

$\mathrm{t} 2=\mathrm{R} 2 * \operatorname{Cos}((\mathrm{ppi}-\mathrm{dcj}) / 2) / \operatorname{Sin}((\mathrm{ppi}-\mathrm{dcj}) / 2)$

angpj1pc $=$ TDraw. Utility. Angle From XAxis $(p j 1, p c)$

Me. hide

And then, the $2 \mathrm{~d}$ graphic of rail and the cross section view of other parts are drafted according to the drawing program's commands below.

Call This Drawing. ModelSpace. Add Line (pc, pd)

Call TDraw. ModelSpace. AddArc (po1, R1, angpo1pd, angpo1pl)

angpo3pq $=$ TDraw. Utility. Angle From XAxis $(p o 3, p q)$

Call TDraw. ModelSpace. AddArc (po3, R2, angpo3pn, angpo3pq)

Call TDraw. ModelSpace. AddArc (po4, R2, angpo3pn, angpo3pq)

Finally, the corresponding regions of rail and sleeper are created and they will be handled by a series of process like stretching, sweeping and duplicating in accordance of these $2 \mathrm{~d}$ graphics. The main program is shown below:

Set objent $1=$ This Drawing. ModelSpace. Add Extruded Soild (objRegion,-height, 0)

Objent1. boolean acUnion, objent1

Dim gcopy (0 To 2) As Acad3DSolid

For $i=0$ To 2

Set ggop (i) = objent1. Copy

Private Sub CommandButton_Click()

End

End Sub

\section{The Addition of Plug-in Menu Based on VBA}

AutoCAD system for the plug-in menu bar shown as Fig.6 below of the ballast track and the ballastless track is reset according to the illustration of track structure. Then the menu bar can call the visual drawing windows by the program written, which is easy for man-machine interaction, and the calling of the $3 \mathrm{~d}$ standard library of the track structure will be more simple, quick and accurate.

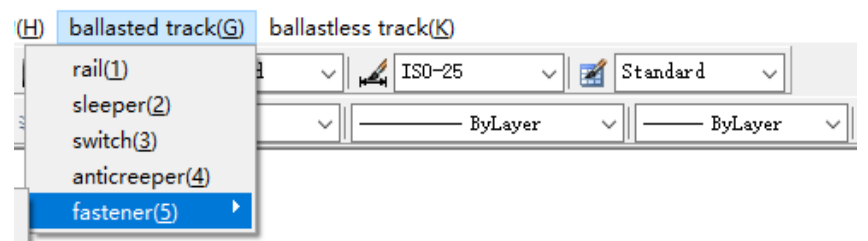

Figure 6. The diagram of custom menu

The format of menu file is .mnu, and the main program of ballast track menu bar is shown below:

// Default AutoCAD NAMESPACE declaration:

$* * *$ MENUGROUP=ACAD2

// Begin AutoCAD Pull-down Menus

$* * * \mathrm{POP} 1$

**FILE

ID_MnFile [ballast $\operatorname{track}(\& I)]$

ID_MnDrawing1 [->rail $(\& 1)]$

ID_MnDrawing2 [-> sleeper( $\& 2)]$

ID_MnDrawing $3[->\operatorname{track} \operatorname{switch}(\& 3)]$

ID_MnDrawing4 [->anti climbing equipment $(\& 4)]$

ID_MnDrawing5 [-> fastening(\&5)]

ID_Audit [slab fastening(\&6)] $\wedge^{\wedge}{ }^{\wedge} c_{-}$vbanz;D;

/VBAmenu/element library.dvb!

ID_Recover [type I fastening $(\& 7) \ldots] \wedge \mathrm{c}^{\wedge} \mathrm{c} \_$vbanz;D; /VBAmenu/element library.dvb!

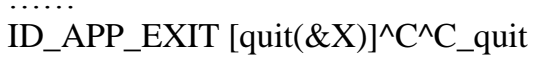

\section{THE Assembling AND THE SHOWING OF THE 3D LIBRARY SOLID}

The visual windows is called directly by custom plug-in menu, which increasing the operability of drawing so as to a more intuitive and quick modeling of track. Taking the $60 \mathrm{~kg} / \mathrm{m}$ ordinary ballast rail and the slab continuous welded rail as examples, corresponding parameters are input after choosing the model,fastening,ballast bed and rail etc. of $3 \mathrm{~d}$ railway switch, and the $3 \mathrm{~d}$ model of railway parts will be created automatically. Then the modeling of the whole railway is finished according to the process of assembling. The result is shown in Fig.7 and Fig.8.

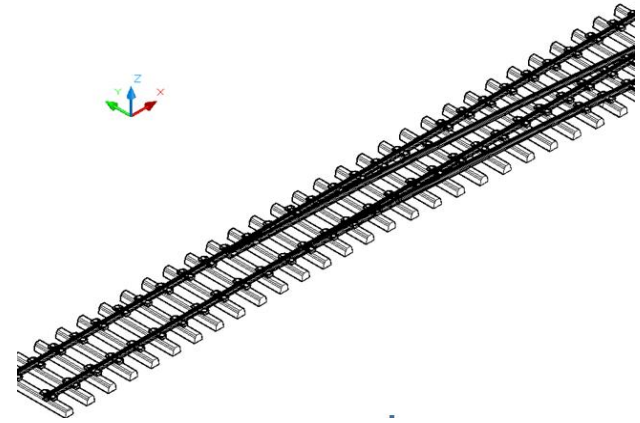

Figure 7. The diagram of building result for ballasted track structure 


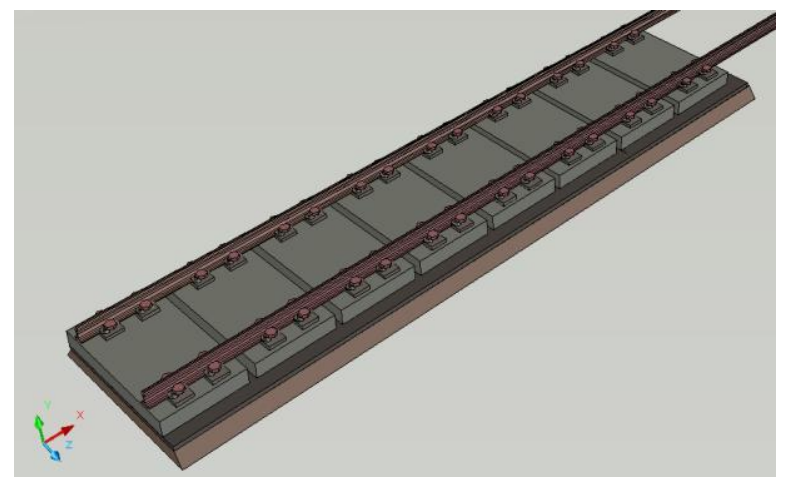

Figure 8. The diagram of building result for ballastless track

\section{CONCLUSION}

The secondary development of AutoCAD based on VBA can realize the functional extendibility of AutoCAD and the parameterized design. This thesis tries to do some related designs and researches on the $3 \mathrm{~d}$ digitized modeling of rail structure by the researching of the characteristic of the rail structure, which is based on VBA IDE on AutoCAD platform. The main conclusions are as follows:

1) The automatic modeling of the present ordinary rail structure is realized, which can be applied to related designs, constructions and learning and is with the trend of the development of railway industry. Besides, it is of considerable realistic significance for creating the $3 \mathrm{~d}$ digitized model library in railway industry.

2) The visual windows and the plug-in menu of the fastening,the sleeper, the rail switch and other parts of rail are designed, which makes the designing of rail structure more intuitive and speedier .It increases the efficiency of modeling and it better meets the requirements of the $3 \mathrm{~d}$ design in the railway.

3) The model proposed in this thesis has a user-friendly interface, and it's simply and rapidly used, besides it's accurate and stable, which provides a reference method and a thought for modeling by VBA in other professions. After the text edit has been completed, the paper is ready for the template. Duplicate the template file by using the Save As command, and use the naming convention prescribed by your conference for the name of your paper. In this newly created file, highlight all of the contents and import your prepared text file. You are now ready to style your paper.

\section{ACKNOWLEDGMENT}

This research was supported by the National Natural Science Foundation of China (51468036), Gansu Natural Science Foundation of China (1310RJZA065); LanZhou JiaoTong University Youth Science Foundation (2013029).

\section{REFERENCES}

[1] Cao Yang, Wang Ping, “ Study on Turnout Linear Design Based on The Method of Plane Parameters, " Railway Engineering, vol. 25, Jan. 2011, pp.101-103.

[2] Zhang Fan, Zheng Likai, "AutoCAD VBA excellent example of curriculum development,” Beijing Tsmghua University Press, 2004.

[3] Kang Feng, Han Feng, "Railway turnout automatic configuration software through VBA,". Railway Computer Application, vol. 24, July. 2015, pp.5-6.

[4] Liu Hou Qiang, Yi Xu Peng, Zhu Cong, "The Application of Three Dimensional Modeling with BIM Technology in Railway Subgrade,". Railway Standard Design, vol. 12, Dec, 2015, pp.20-22.

[5] Alwisy, "BIM Approach for Automated Drafting and Design for Modular Construction Manufacturing," Journal of Computing in Civil Engineering, vol. 13, Feb. 2012, pp.34-37.

[6] Li Changxun, "AutoCAD VBA program development technology," Beijing Defence Industry Press, 2004.

[7] Shen Qizhi, Ning Ailin, "The development of standard parts graphics library based on AutoCAD's VBA," Journal of Shaoyang College, vol.14, Apr.2001, pp.248-249.

[8] Guo H L, LI H, SKITMORE M, "Life cycle management of construction projects based on Virtual Prototyping technology," Journal of Management in Engineering, vol.26, Jan. 2010, pp.41-47.

[9] Brito A C, Oliveira C D, Marques P S, "A simulation study of a multi-site production plant using arena," European Simulation and Modelling Conference. 2011.

[10] Wang Xi, Luo lei, "The research and discussion in the field of architectural design on AutoCAD," Housing and Real Estate, vol.8, Jan. 2015, pp.136-136.

[11] Ding Hangxing, Ren Fengyu, Wang Chunxian, "Automatic Generation of Coordinate Parameter Table of Roadway in AutoCAD Environment," Journal of Northeastern University, vol.36, Jun, 2015, pp.872-875. 